\title{
The Research about Collaboration Techniques for Aerial and Ground Mobile Robots
}

\section{Dunhao Liu ${ }^{12}$}

Hubei Provincial Key Labratory of Intelligent Robot, 430205, Wuhan, China

E-mail: 850114116@qq.com

\section{Xun $\mathbf{L i}^{3}$}

Hubei Provincial Key Labratory of Intelligent Robot, 430205, Wuhan, China

E-mail:30718736@qq. com

\section{Wei Liu}

Hubei Provincial Key Labratory of Intelligent Robot, 430205, Wuhan, China

E-mail: Iiuwei@wit.edu.cn

With the development of robotics technology, people show great interest in the research on the cooperation between aerial and ground robots. The collaborative technologies not only expand the robots application range, but also improve their tasking ability in reconnaissance, surveillance and inspection domains. This paper makes a statement in terms of four core techniques in the course of cooperation, namely, the system architecture, the mapping, the localization and the navigation, then makes a brief analysis of the application scenarios, and finally makes the conclusion.

CENet2015

12-13 September 2015

Shanghai, China

\section{${ }^{1}$ Speaker}

${ }^{2}$ Correspongding Author

${ }^{3}$ This paper is the start of our robots cooperation project which comes from Nature Science Foundation of Hubei Province(No.2014CFB779), and the future work will be the experiments and algorithm designed. 


\section{Introduction}

In recent years, a number of research communities and enterprises show great interests in autonomous flying robots. Flying platforms with low price and small volume have become more and more general and the payload allows them equipped with some basic equipment, such as a variety of sensors and cameras, etc. The increasing mobility of which also extends their applications.

Nowadays, applications of UAV(Unmanned Aerial Vehicle) in the UAV study field have become a hot topic. The major applications in UAV combine UAV and sensors, which has incarnated a wide range of perception capability and applications such as reconnaissance, surveillance and inspection, etc. It features such advantages as small volume, flexible mobility and broad view field, which indeed benefits us a lot; nevertheless, the small volume lead to the shortcoming of poor load and weak endurance is especially prominent. In this case, the advantages of cooperation between aerial robot and ground robot are well represented. Ground robots can not only be the basic platform for aerial robots but also a load carrying. The aerial robot utilizes its broad perspective to provide real-time ground information for the ground robots. As they execute mission collaboratively after message processing and decision-making, they can not only improve the rate and efficiency of task completion, but also perform complex tasks that single robot cannot complete. Various experimental platforms are shown as below:
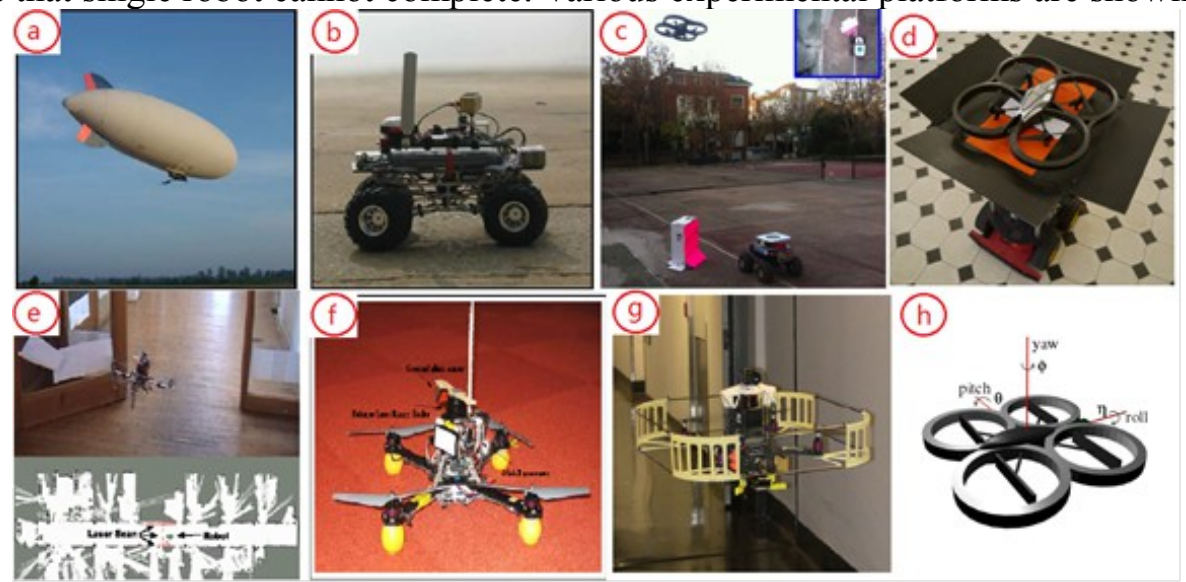

Figure 1: Various experimental platforms. (a) The blimp. (b) The ground. (c) The aerial and ground robot. (d) The aerial and ground robot. (e) The aerial robot. (f) The aerial robot. (g) The aerial robot. (h) The aerial module

\section{Collaboration Techniques for Aerial and Ground Mobile Robots}

The cooperation between aerial robot and ground robot usually utilizes broad perspective of aerial robot to capture images, which are generally processed by three ways: 1 . transferring images to the ground station and using the ground station processor unit to process images; 2 . the aerial robot incorporates a processor unit to possess images directly and output the processing result; 3 . sending images to the ground robot, which fuses the processing result with its own sensor information to get desired outputs after image processing. The outputs can be used to build map, locate position, ovoid obstacles, plan and control path, etc. The following parts show the detail demonstration of system architecture, mapping, locating, control and navigation. A typical structure for the collaboration techniques can be summarized as below. 


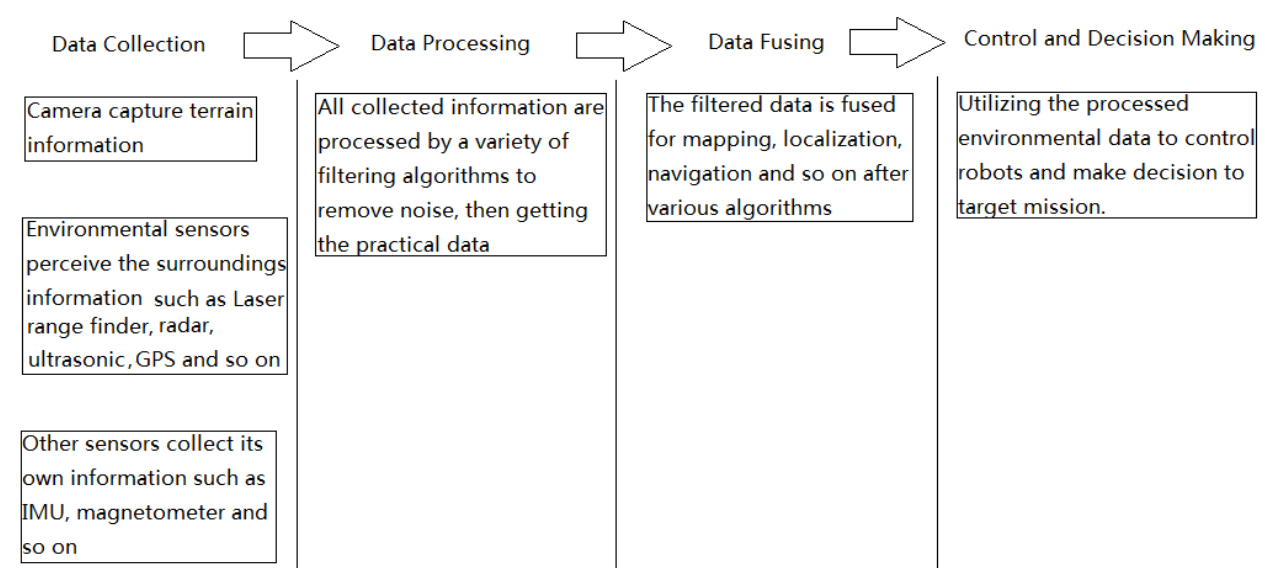

Figure 2: The typical structure is constructed by data collection, data process, data fusing, control and decision making.

\subsection{Architecture for Multi-Robot Systems}

The limitation of perception and decision-making ability for single robot results in its low capability of completing easy tasks or incapability of completing some complex tasks. In multirobot systems, as information is shared among robots, complex tasks are rational planned and allocated, the robots collectively complete a complex task that a single robot is unable to complete.

The author introduced the multi-robot systems[1], the most common of which can be summarized as centralized, hierarchical, decentralized and hybrid, DIRA(Distributed Robot Architecture) was developed from a hybrid architecture and the Nerd Herd firstly studied social behaviours in multi-robot teams as well as the alliance architecture[2-3], another research on multi-robot team architecture in early period[4].

The authors utilized AGC(Air-Ground-Cooperation) for target searching missions[5]. Their research the architecture was composed of three layers: the high altitude for decision making, used to determine the movement of ground robots according to the target; the middle altitude for tracking as well as maintaining the ground robots in its own field; the low altitude for mission. The ground robot executed the exploration mission based on the waypoints.

The authors used ROS middle-ware framework to tackle the inter-process synchronous communication[6-7]. In terms of other architecture such as modular architecture[8], various components rely on the publish-subscribe mechanism to communication via network, the timecritical modules with device drivers are executed on-board, and other algorithms which are computationally intensive for localization and mapping with the UI for users are executed on a remote computer.

\subsection{Mapping}

The authors researched the navigation and obstacle mapping system for aerial and ground robots in large outdoor environment[6]. The navigation system for localization and obstacle detection techniques utilize relative and absolute sensors. Relative sensors are internal to robot to measure another state or position. Absolute sensor measures an external or global reference. The system gets the images through the aerial robot, then fuses the information with the position absolute to the ground robot to acquire the absolute position of the obstacles, thus completes the obstacle mapping upon mapping algorithm. The experiment results and data are shown as below. 


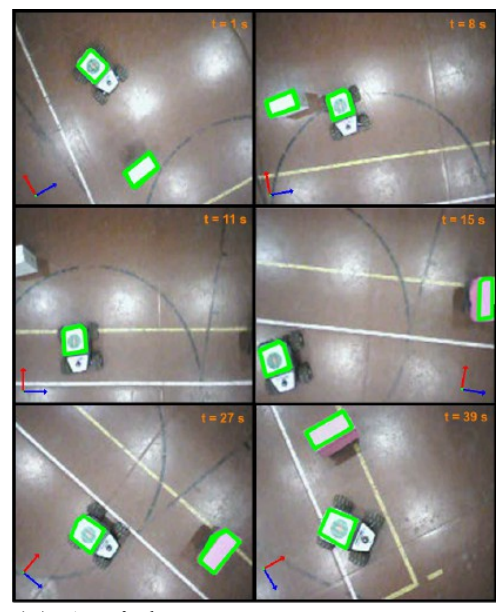

(a) Aerial Images

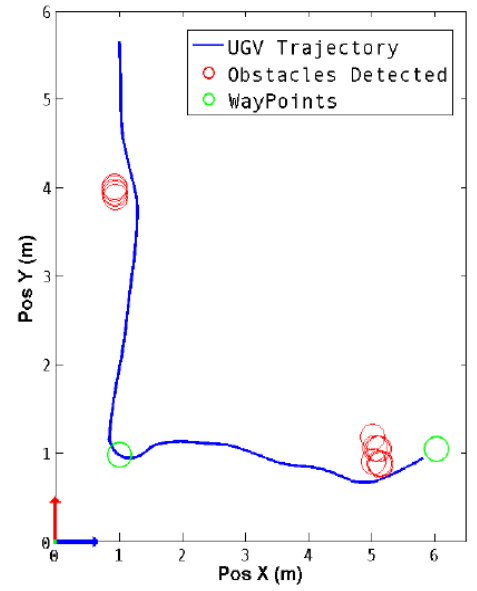

(b) Trajectory

Figure 3: (a) Images acquired from the UAV when executing the obstacle avoidance operation. The red arrow is for $\mathrm{X}$ axes and the blue arrow is for Y axes. (b) The blue line shows the UGV trajectory which is obtained from the EKF.

\begin{tabular}{|l|c|c|c|c|}
\hline & mean pos. X (m) & std. dev. X & mean pos. Y (m) & std. dev. Y \\
\hline Obstacle1 & 0.9896 & 0.0194 & 4.1373 & 0.0468 \\
\hline Obstacle2 & 5.0930 & 0.1143 & 1.1158 & 0.05 \\
\hline
\end{tabular}

Table 1: Mean Values with Standard Deviation Found for the Obstacles in the Trajectory.

Other authors regarded the aerial robot as the remote sensor[9-10], formatted the threedimensional geometric image as the map to guide the ground robot navigating and obstacle avoiding after image processing. The authors mapped with the loop closure[6]. Of course, there were many researches for instance, the authors studied the trajectory tracking with pre-defined $\operatorname{maps}[11-12]$.

\subsection{Localization}

The localization needs to know the exact location of the robot. A variety of environmental sensors such as sonar ranging[13], images[14] and laser ranging[15] for the robot position estimation are used for environment features, feature-based approaches[16-17], and IPC(Iterative Closest Point) algorithm, which are all getting data from the environment feature and then estimating the specific position to complete the localization. Bayesian statistical analysis is one of the most robust techniques for localization[13], in which a feasibility $\mathrm{s}_{\mathrm{t}}$ at time $t$ is inferred from following series of noisy actions $u_{1: t}$ as well as the measurement $z_{1: t}$. With some standard assumptions about the actions and observations, the posterior belief can be summarized as

$$
p\left(s_{t} \mid u_{1: t}, z_{1: t}\right)=\frac{1}{z} p\left(z_{t} \mid s_{t}\right) \int_{s} p\left(s_{t} \mid u_{t}, s_{t-1}\right) p\left(s_{t-1}\right) d s_{t-1}
$$

$\mathrm{Z}$ is the normalization factor and Equation (1) can be called as the Bayes' Filter Equation, which updates the state distribution with efficient recursion.

The feature localization is posed as a linearized Gaussian estimation problem[18], the Kalman Filter contains an information state vector $\hat{v_{f, i}}(k \mid k)$ as well as the matrix $M_{f, i}(k \mid k)$ that discriminated by subscript $f$ for each feature $i$, which relates to the covariance and feature estimate mean by

$$
\begin{array}{r}
\hat{v_{f, i}}(k \mid k)=P_{f, j}^{-1}(k \mid k) \hat{x}(k \mid k) \\
M_{f, i}(k \mid k)=P_{f, i}^{-1}(k \mid k)
\end{array}
$$


Each sensor measurements $\mathrm{z}$ contributes to an information vector as well as a matrix which captures the covariance of observation and the mean is similar to $P\left(z_{s}(k) \mid x\right) \sim N\left(\mu_{s}\right.$, $\left.\Sigma_{s}\right)$.

$$
i_{f, s}(k)=\sum_{s}^{-1}(k) u_{s}(k), I_{f, s}(k)=\sum_{s}^{-1}(k)
$$

After fusing with the accumulated prior information, we can summrise the formula as

$$
\begin{gathered}
\hat{v_{f, i}}(k \mid k)=\hat{v_{f, i}}(k \mid k-1)+\sum_{s} i_{f, s}(k) \\
M_{f, i}(k \mid k)=M_{f, i}(k \mid k-1)+\sum_{s} I_{f, s}(k)
\end{gathered}
$$

from which, the state estimate can be recovered.

The localization approach was provided by setting landmarks in a special terrain, then fusing the inherent sensor data in robot to get exactly position and orientation as well as other required information[18-19]. At last, the authors compared differences among waypoint, GPS/IMU approach, GPS for ground location and draw only to find that the localization should be the key technique and a good coordination mechanism was the basic element in cooperation.

The position estimation fused the odometry, IMU and GPS and used an EKF (Extended Kalman Filter) for position estimation in the global coordination frame of ground robot, then used another $\mathrm{KF}$ (Kalman Filter) for the position estimation of obstacle after the image processing algorithm, thus completed the location of the ground robot and the obstacle[6].

The authors used colour pattern, UGV (Unmanned Ground Vehicle) equipped with all necessary sensors with a high capacity power; and the UGV equipped with a helipad can also act as a carrier for the UAV(Unmanned Aerial Vehicle). It can be used for defining the origin as well as the orientation for the UAV as well, then estimate its position in combination of the dead reckoning and visual tracking[20].

Data collected from sensors mostly have to get through the filtering algorithm to remove noise. Kalman filter and other converted Kalman filters perform well in this regard. The authors made a simply treatment for localization through special terrain and colour pattern[19-20], but the real environment can be rather complex. Feature-based approaches performed well in SLAM (Simultaneous Localization and Mapping), but both were too strenuous for the onboard processor; but the IPC algorithm may easy run at $20 \mathrm{~Hz}$ on the onboard processor[16-17].

\subsection{Navigation}

Navigation is the method which guides a device to move from one specified waypoint to another waypoint; however, the aerial and ground robot collaborative navigation can be described as: the aerial robot utilizing its broad perspective to provide image information for the ground robot under different circumstances to assist the ground robot to move from origin to the target position, or the UAV/UGV automatic navigation in the course of collaborative mission execution. A series of questions which need to be addressed during navigating can be summarized as the processes ranging from state estimation to trajectory planning.

The air-ground coordination scenario was a remote reconnaissance mission[19]. The robots team was composed of a group of ground vehicles and a blimp which was dispatched to autonomously map an urban area. The blimp was fully autonomous which can also transmit digital images to the operator and the images that can be used to map for ground vehicles in its field to locate the vehicles. Even without GPS, the operator can also offer navigation for ground robots to move to specified position.

The study of aerial and ground robot cooperative navigation outdoors without knowing the environment as well as the map[6]. The UGV is regarded as the platform for UAV to take off and land; besides, UGV is designed with a polygon, which is easy for features extraction, and the colour pattern is also easy to distinguish the UGV from the ground. UAV takes images and sends them to the ground station for the recognition of UGV after features extraction algorithm. If the UGV encounters an obstacle during the navigation, the station still utilizes the features 
extraction algorithm to recognize the obstacle, then avoid such obstacle through the Steering Behaviors[21].

The authors described the heterogeneous robot system to solve missions of regular monitoring indoors cooperatively[20]. UAV would be equipped with two cameras used to inspect the place where UGV cannot arrive. UGV equipped with a platform is used for automatic landing when UAV encounters the obstacle and cannot pass through or is of low power. UGV can also charge UAV as well as all required sensors and high-capacity power source. As described by test scenario, the robotic team visits a series of places of interest periodically, as pre-defined by experimenters. UGV passes through the pre-defined path to scan areas of interest by its sensor. If the area is unreachable, the UAV will automatic tack off, then fly to the detection area and take some images, then fly back to the UGV and land. They will continue to the next place of interest upon completion of the current detection mission.

The navigation can be divide into autonomous navigation and non-autonomous navigation based on whether someone is participated in the process. To some extent, it reflects the intelligence during navigation. The higher level of the autonomy is, the more complex of the system will be. The authors implemented the UVA autonomous navigation during collaborative tasks[20]. UAV was able to take off automatically and fly to the specific area, then return back and land after capturing the image of the inspected area, unreachable bt UGV. The operator fully controlled UAV under the condition of non-autonomous navigation[22]. The experimenter controlled UVA to keep UGV in its field of view without knowing the map, then the experimenter selected the waypoints after receiving images as captured by UAV to guide UGV to steer and avoid obstacles.

\section{Application Scenarios}

Deploy the aerial and ground robot in inhospitable workplaces to perform repetitive, tiresome tasks as the surveillance mission[20], or performing reconnaissance mission in an unknown territory where it may be dangerous and unsuitable for UGV to pass through without knowing the condition, thus UAVs are able to survey the surrounding area and provide early warning as the reconnaissance missions[23], or detect the specific area where humans may be exposed to danger in case operation in hostile environments, thus an union detecting team composed of UAVs and UGVs is deployed in the detected area, the aerial robots fly along a trajectory and uniformly scan the regions to locate the target[24], or the collaborative tracking control[25], or the searching and rescuing missions[26], or the inspection tasks.

\section{Conclusion}

The cooperation between aerial and ground robots is a new area of research. As the capacity of a single aerial robot is limited, the ability of performing tasks can be improved without limitation by means of collaboration with the ground robot. The potential of cooperation between aerial and ground robot is represented from the immeasurable prospects, whatever in the military reconnaissance, surveillance, inspection domains or the civilian rescue, security, patrol domains. Collaboration techniques embody the intelligence of robot and the intelligence of robot largely depends on the accuracy of sensors, such as when using the distance sensor, the measurement is better, the treatment effect of algorithm is better, the GPS positioning and the trajectory planning can be more accurate; besides, in the domain of image recognition and processing, as the map understanding will directly affect the robot's control, the mapping technology both in plane and three-dimensional space also requires continuous improvement. In all, further study on the coordination technology is not only to get a very broad application prospects in military and civilian fields, but also develop basic technique of modern robotic systems in a more intelligent and advanced manner. 


\section{References}

[1] L.E. Parker. Multiple Mobile Robot Systems[C]. Springer Handbook of Robotics. Springer Berlin Heidelberg. pp, 921-941(2008)

[2] R. Simmons, S. Singh, D. Hershberger, J. Ramos, T. Smith. First Results in the Coordination of Heterogeneous Robots for Large-Scale Assembly[M]. Experimental Robotics VII. Springer Berlin Heidelberg. pp, 323-332(2001)

[3] M.J. Matari'c. Issues and Approaches in the Design of Collective Autonomous Agents[J]. Robotics and Autonomous Systems. 16(2), pp, 321-331(1995)

[4] L.E. Parker. ALLIANCE: An Architecture for Fault-Tolerant Multi-Robot Cooperation[J]. Robotics and Automation, IEEE Transactions on. 14(2), pp, 220-240(1998)

[5] L. Chaimowicz, V. Kumar. A. Framework for the Scalable Control of Swarms of Unmanned Ground Vehicles with Unmanned Aerial Vehicles[C]. The 10th International Conference on Robotics and Remote Systems for Hazardous Environments(2004)

[6] S.J. Shen, N. Michael, V. Kumar. Autonomous Multi-Floor Indoor Navigation with a Computationally Constrained MAV[C]. IEEE International Conference on Robotics and Automation. IEEE Conference Publications. pp. 20-25(2011)

[7] M. Garzón Oviedo, J.R. Pereira Valente, D. Zapata, R. Chill, A.B. Cruz. Towards a Ground Navigation System Based in Visual Feedback Provided by a Mini UAV[C]. Proceedings of the IEEE Intelligent Vehicles Symposium Workshops. Alcal de Henares, Spain. Vol.37(2012)

[8] S. Grzonka, G. Grisetti, W. Burgard. Towards a Navigation System for Autonomous Indoor Flying[C]. Robotics and Automation, 2009. ICRA'09. IEEE International Conference on. IEEE, pp, 2878-2883(2009)

[9] T. Stentz, A. Kelly, P. Rander. Integrated Air/Ground Vehicle System for Semi-Autonomous OffRoad Navigation[J]. Robotics Institute, Vol.18(2002)

[10] A. Stentz, A .Kelly, P. Rander, H. Herman, O. Amidi. Real-Time, Multi-Perspective Perception for Unmanned Ground Vehicles[C]. Proc. of the AUVSI Unmanned Systems Conference(2003)

[11] J. Ghommam, H. Mehrjerdi, M. Saad, F. Mnif. Formation Path Following Control of Unicycletype Mobile Robots[J]. Robotics and Autonomous Systems. 58(5), pp, 727-736(2010)

[12] H.S. Ramiandrez, R.C. Linares. Trajectory Tracking for Non-Holonomic Cars: A Linear Approach to Controlled Leader-Follower Formation[C]. IEEE Conf. on Decision and Control (CDC). 49th IEEE Conference on. IEEE, 2010, pp, 546-551(2010)

[13] J. Leonard, H.D. Whyte. Mobile Robot Localization by Tracking Geometric Beacons[J]. IEEE Transactions on Robotics and Automation. IEEE, 1991, 7(3), pp, 376-382(1991)

[14] S.Thrun, D. Fox, W. Burgard, F. Dellaert. Robust Monte Carlo Localization for Mobile Robots[J]. Artificial Intelligence. 128(1-2), pp, 99-141(2000)

[15] D. Gurdan, J. Stumpf, M. Achtelik, K.M. Doth, G Hirzinger, D. Rus. Energy-Efficient Autonomous Four-Rotor Flying Robot Controlled at $1 \mathrm{khz}[\mathrm{C}]$. Robotics and Automation, 2007 IEEE International Conference on. IEEE, 2007, pp, 361-366(2007)

[16] G.D. Tipaldi, K.O. Arras. FLIRT-Interest Regions for 2D Range Data[C]. Robotics and Automation (ICRA), 2010 IEEE International Conference on. IEEE, 2010, pp, 3616-3622(2010)

[17] F. Ramos, D. Fox, H.D Whyte. C.R. F-Matching: Conditional Random Fields for Feature-Based Scan Matching[C]. Robotics: Science and Systems(2007) 
[18] B. Grocholsky, A. Makarenko, T. Kaupp, H. F. Durrant-Whyte. Scalable Control of Decentralised Sensor Platforms[M]. Information Processing in Sensor Networks: 2nd Int Workshop, IPSN03, pp, 96-112(2003)

[19] L .Chaimowicz, B. Grocholsky, J. Keller, V. Kumar, C.J. Taylor. Experiments in Multirobot AirGround Coordination[C]. Robotics and Automation. Proceedings. ICRA'04. 2004 IEEE International Conference on. Vol.4, pp, 4053-4058(2004)

[20] M.K. Habib, Y. Baudoin, F. Nagata. Robotics for Rescue and Risky Intervention[C]. IECON 2011-37th Annual Conference on IEEE Industrial Electronics Society. IEEE, 2011, pp, 3305 3310(2011)

[21] C.W. Reynolds. Steering Behaviors for Autonomous Characters[C]. Proceedings of Game Developers Conference, San Jose, CA, USA, pp.763-782(1999)

[22] E. H. C. Harik, F. Guérin, F. Guinand, J. F. Brethé, H. Pelvillain. A Decentralized Interactive Architecture for Aerial and Ground Mobile Robots Cooperation[C]. Control, Automation and Robotics (ICCAR), 2015 International Conference on. IEEE, 2015, pp, 37-43(2015)

[23] H.G. Tanner, D.K. Christodoulakis. Cooperation between Aerial and Ground Vehicle Groups for Reconnaissance Missions[C]. Decision and Control, 45th IEEE Conference on. IEEE, 2006, pp, 5918-5923(2006)

[24]H.G. Tanner. Switched Uav-Ugv Cooperation Scheme for Target Detection[C]. Robotics and Automation, IEEE International Conference on, IEEE, 2007, pp, 3457-3462(2007)

[25] J.Y. Choi, S.G. Kim. Collaborative Tracking Control of UAV-UGV[J]. World Academy of Science, Engineering \& Technology. Issue 71, pp, 1430(2012)

[26] K. Sugihara, I. Suzuki. Distributed Algorithms for Formation of Goemetric Patterns with Many Mobile Robots[J]. Journal of Robotic Systems. 13(3), pp, 127-139(1996) 